\title{
Tumor downstaging in muscle invasive bladder cancer with neoadjuvant systemic therapy-does it lead to prolonged survival?
}

\author{
Isamu Tachibana, Hristos Z. Kaimakliotis \\ Department of Urology, Indiana University School of Medicine, Indianapolis, IN, USA \\ Correspondence to: Hristos Kaimakliotis. 535 N Barnhill Dr. Suite 150, Indianapolis, IN 46202, USA. Email: hkaimakl@iupui.edu. \\ Provenance and Peer Review: This article is commissioned and reviewed by the Section Editor Dr. Xiao Li (Department of Urology, Jiangsu Cancer \\ Hospital \& Jiangsu Institute of Cancer Research \& Affiliated Cancer Hospital of Nanjing Medical University, Nanjing, China). \\ Comment on: Martini A, Jia R, Ferket BS, et al. Tumor downstaging as an intermediate endpoint to assess the activity of neoadjuvant systemic therapy \\ in patients with muscle-invasive bladder cancer. Cancer 2019;125:3155-63.
}

Submitted Sep 02, 2019. Accepted for publication Dec 20, 2019.

doi: $10.21037 /$ tau.2019.12.28

View this article at: http://dx.doi.org/10.21037/tau.2019.12.28

It has been firmly established that cisplatin-based chemotherapy in the neoadjuvant setting prior to radical cystectomy improves overall survival in patients with muscle invasive bladder cancer (1). This study demonstrates and argues that improvement in overall survival with neoadjuvant chemotherapy (NACT) is not only related to a complete clinical response (pT0N0), but also to any pathologic downstaging, whether complete or a partial response from the initial clinical stage at diagnosis (2). Both the Retrospective International Study of Cancer of the Urothelial Tract (RISC) and National Cancer Database (NCDB) were queried in this study to identify a cohort of patients that had complete pathologic response or downstaging with NACT. Multivariable Cox proportional hazard models were used to demonstrate the effect of downstaging and complete pathologic response on overall survival in the data set. The analysis ultimately found that any pathologic response is associated with improved overall survival, but a couple caveats should be noted.

First, data compiled for the study includes patients from the NCDB with T2-T4N0M0 disease, which may make it difficult to accurately and consistently determine clinical staging given that $\mathrm{T} 3 / \mathrm{T} 4$ staging relies on imaging interpretation and bimanual examination. Both of which are highly operator dependent and a bimanual examination under anesthesia is rarely performed and well-documented in the pre-operative setting. Nonetheless, despite the limitation in accurately determining pre-operative clinical staging, where such clinical staging is indeed available, the results and conclusions should generally remain the samethat any pathological response and downstaging is a marker for improved survival.

Another limitation to note in this accumulated patient population is that clinical downstaging and complete response may not only be the result of NACT but also as result of a well-performed TURBT. As mentioned in the study, one of the confounding factors during the work-up for bladder cancer is that transurethral resection alone can clinically downstage patients. As reported, in this article, Shariat et al. reported a rate of $22 \%$ clinical downstaging for patient undergoing cystectomy alone (3). Brant et al. demonstrated that clinical downstaging may be impacted by TURBT alone about $38 \%$ of the time, but still showed that patients receiving NACT showed a greater response to treatment (4). A well performed, TURBT without NACT may address small volume cT2 disease, but will not account for residual T3/4 or nodal involvement. The authors control for this in their study.

Ultimately, this study makes the point that overall survival is correlated by the different levels of response to NACT, which should then be considered as a valid primary or secondary outcome in clinical trials with promising novel agents that have shown success in early phase trials. Based on the results of this study, we can cautiously infer that the initial success of novel agents such as immune checkpoint inhibitors (PD-1 and PD-L1 inhibitors for urothelial carcinoma) demonstrating preliminary results of disease regression should lead to favorable outcomes and increased 
overall survival after appropriate follow up. Of note, based on this study $33-35 \%$ of patients achieved pathologic down staging and complete pathologic response was achieved by $15-20 \%$ of the patients based on the cohorts from the two databases. In a recent neoadjuvant clinical trial where patients received pembrolizumab followed by cystectomy, $42 \%$ of patients experienced a complete pathologic response and 54\% of patients experienced clinical downstaging (5). Another combined neoadjuvant chemo-immunotherapy trial showed an unprecedented $48 \%$ complete pathologic response and $65 \%$ clinical downstaging to combination cisplatin, gemcitabine and pembrolizumab (6). Both studies classified pathologic downstaging as defined by any pathology $\mathrm{T} 2$ or less in the clinical trial involving pembrolizumab including patients with complete response, whereas this study defined downstaging as any patient who experienced a response to NACT regardless of staging. Perhaps this new measure of outcome would lead to an even higher "downstaging" effect, which will hopefully correlate to improved survival. Long-term follow-up from these two clinical trials will help answer this and potentially validate this study by Martini et al.

The authors further argue, that given this improvement in overall survival based on any degree of pathologic downstaging, perhaps we can measure success differently in clinical trials going forward. As clinical trials for immune checkpoint inhibitors are completed, pathological response should correlate to longitudinal improvement in overall survival. However, only after longer follow up will we be able to confirm that immune checkpoint inhibitors can lead to longer overall survival and utilize any pathologic downstaging as a functional measure of improvement of long-term survival benefit. There is still the possibility that the overall survival curve may not exactly produce the same survivability curves as NACT. The differences in the biology and pharmacology between immune checkpoint inhibitors and NACT can change the survivability curve despite initial results of disease regression. To illustrate these differences, some studies have shown that pembrolizumab or atezolizumab can prolong survival and have efficacy in the adjuvant setting after failing cisplatin based chemotherapy $(7,8)$. Patients receiving PD-1 inhibitors have shown evidence of developing adaptive mechanism for immune resistance (5). If patients experience disease progression after receiving a neoadjuvant checkpoint inhibitor, the biology of this advanced urothelial carcinoma may be different compared to bladder cancer that is naïve to a checkpoint inhibitor. It is unclear exactly what the longitudinal survival curves will look like in patients who received these $\mathrm{PD}-1$ inhibitors. Thus, the comparative analysis looking at cancer free survival and overall survival with patients receiving PD-1 and PD-L1 inhibitors and patients receiving NACT will be needed.

In the end, despite convincing evidence that any pathological response leads to improved overall survival, in the setting of NACT and cystectomy, making a similar conclusion with novel therapies is not absolute. Although demonstrating disease regression or complete response shows promise in terms of overall measurable survival, confirmation of efficacy is yet to be definitively determined until enough time passes for appropriate follow up of these patients.

\section{Acknowledgments}

Funding: None.

\section{Footnote}

Conflicts of Interest: All authors have completed the ICMJE uniform disclosure form (available at http://dx.doi. org/10.21037/tau.2019.12.28). The authors have no conflicts of interest to declare.

Ethical Statement: The authors are accountable for all aspects of the work in ensuring that questions related to the accuracy or integrity of any part of the work are appropriately investigated and resolved.

Open Access Statement: This is an Open Access article distributed in accordance with the Creative Commons Attribution-NonCommercial-NoDerivs 4.0 International License (CC BY-NC-ND 4.0), which permits the noncommercial replication and distribution of the article with the strict proviso that no changes or edits are made and the original work is properly cited (including links to both the formal publication through the relevant DOI and the license). See: https://creativecommons.org/licenses/by-nc$\mathrm{nd} / 4.0 \%$.

\section{References}

1. Grossman HB, Natale RB, Tangen CM, et al. Neoadjuvant chemotherapy plus cystectomy compared with cystectomy alone for locally advanced bladder cancer. $\mathrm{N}$ Engl J Med 2003;349:859-66. 
2. Martini A, Jia R, Ferket BS, et al. Tumor downstaging as an intermediate endpoint to assess the activity of neoadjuvant systemic therapy in patients with muscleinvasive bladder cancer. Cancer 2019;125:3155-63.

3. Shariat SF, Palapattu GS, Karakiewicz PI, et al. Discrepancy between clinical and pathologic stage: impact on prognosis after radical cystectomy. Eur Urol 2007;51:137-49; discussion 149-51.

4. Brant A, Kates M, Chappidi MR, et al. Pathologic response in patients receiving neoadjuvant chemotherapy for muscle-invasive bladder cancer: Is therapeutic effect owing to chemotherapy or TURBT? Urol Oncol 2017;35:34. e17-34.e25.

5. Necchi A, Anichini A, Raggi D, et al. Pembrolizumab as Neoadjuvant Therapy Before Radical Cystectomy in Patients With Muscle-Invasive Urothelial Bladder Carcinoma (PURE-01): An Open-Label, Single-Arm,

Cite this article as: Tachibana I, Kaimakliotis HZ. Tumor downstaging in muscle invasive bladder cancer with neoadjuvant systemic therapy-does it lead to prolonged survival? Transl Androl Urol 2020;9(2):831-833. doi: 10.21037/tau.2019.12.28
Phase II Study. J Clin Oncol 2018:36. doi: 10.1200/ JCO.18.01148.

6. Hoimes CJ, Albany C, Hoffman-Censits J, et al. A phase $\mathrm{Ib} / \mathrm{II}$ study of neoadjuvant pembrolizumab (pembro) and chemotherapy for locally advanced urothelial cancer (UC). Ann Oncol 2018;29:mdy424.039.

7. Narayan V, Kahlmeyer A, Dahm P, et al. Pembrolizumab monotherapy versus chemotherapy for treatment of advanced urothelial carcinoma with disease progression during or following platinum-containing chemotherapy. A Cochrane Rapid Review. Cochrane Database Syst Rev 2018;7:CD012838.

8. Powles T, Duran I, van der Heijden MS, et al. Atezolizumab versus chemotherapy in patients with platinum-treated locally advanced or metastatic urothelial carcinoma (IMvigor211): a multicentre, open-label, phase 3 randomised controlled trial. Lancet 2018;391:748-57. 